\title{
Le corps de la honte
}

Par ALIEVTINA HERVY

Université de Liège

Résumé En prenant appui sur les analyses consacrées par Sartre au regard d'autrui dans L'Être et le Néant, nous nous proposons dans ce qui suit d'appréhender le phénomène de la honte comme un phénomène éminemment social où le rapport au corps vécu se trouve singulièrement mis en jeu. Pour ce faire, sont mobilisés, entre autres, les travaux de Levinas, de Derrida, d'Agamben et les descriptions d'Annie Ernaux dans son roman intitulé $L a$ Honte. Nous tentons de montrer que mettre en exergue le caractère social de la honte suggère de soustraire ce phénomène à un cadre moral étroit, qui le réduit à la seule expression de la faute ou de la culpabilité individuelle. Ce faisant, un des enjeux de notre propos consiste à mettre l'accent sur la manière dont le rapport vécu au corps cristallise les projections imaginaires et les significations sociales inhérentes à l'expérience de la honte. En dernière étape de ce parcours, les descriptions de Michel Foucault sur « Le corps utopique » nous amènent à considérer le pouvoir spatialisant des corps comme un pouvoir utopisant. En ce sens, elles permettent ainsi d'ouvrir une réflexion sur l'impact et les limites d'une captation des corps par les significations sociales et imaginaires à travers lesquelles les relations intersubjectives se façonnent et se déploient.

\section{Introduction}

Notre réflexion prend comme point de départ la question suivante : imaginer, est-ce s'évader et vivre dans un monde où le corps serait totalement absent ? Que dire de ce corps, lorsque nous imaginons les personnages du roman que nous lisons; où se trouve-t-il ? Il semble pourtant bien « là », enfoncé dans le fauteuil, soutenant la lecture au cours de laquelle il apparait pourtant comme 
neutralisé, le sujet imaginant étant tout entier tourné vers le déroulement des actions et des événements de la fiction. Il semble que lorsque nous nous posons la question "qu'est-ce qu'imaginer? ", c'est presque naturellement que nous « biffons », « neutralisons » le corps, notre corps, comme s'il n'en était pas un acteur majeur, mais un simple support pour effectuer le saut nécessaire afin de se transporter vers un là-bas imaginaire. Et pourtant, en lisant les nombreux textes que Husserl a consacrés à l'imagination, qu'il s'agisse de la conscience d'une image (Bildbewusstsein) ou de la Phantasie, cette question se trouve thématisée par leur auteur, qui évoque un dédoublement, une scission de l'ego (Spaltung), comme si celui-ci pouvait en même temps - mais selon des modalités très spécifiques - être «ici » et « là-bas $»^{1}$. Comment décrire ce corps, le nôtre, qui se trouve impliqué dans l'imagination, tout en étant à la fois suspendu par elle ? Comment rendre compte de ce caractère énigmatique du corps qui, à travers sa mise en veille même, se projette sous un mode fantasmé dans le monde de la phantasia ? Cette question des rapports entre corps et imagination est importante selon nous, car elle suggère de considérer autrement l'imagination que comme une activité purement mentale, désincarnée, où le sujet se trouve comme en position de surplomb par rapport à ce qu'il imagine, comme s'il pouvait s'y rendre et s'en extraire à loisir. Plus particulièrement, il s'agit non seulement, en posant cette question, de s'interroger sur les rapports étroits qui lient l'affectivité et l'imagination ${ }^{2}$ mais, plus largement, de re-contextualiser l'imagination au sein des conditions sociales et intersubjectives qui impactent en profondeur son déploiement. C'est en ce sens que nous avons choisi de nous intéresser au phénomène de la honte, qui permet d'explorer de quelle façon peuvent se cristalliser dans les corps toute une série de projections imaginaires et, à travers elles, les significations sociales, les valeurs et les normes qui les accompagnent.

Lorsque l'on évoque la honte, il semble que ce phénomène soit à première vue indissociable d'un cadre moral. En effet, à leur lecture, il semble que les analyses de Sartre, de Derrida, de Levinas et d'Agamben demeurent d'une manière ou d'une autre hantées par le spectre de la

${ }^{1}$ Cf. E. Husserl, Phantasia, conscience d'image, souvenir. De la phénoménologie des présentifications intuitives. Textes posthumes (1898-1925), trad. fr. R. Kassis \& J.-F. Pestureau, Grenoble, éditions Jérôme Millon, coll. « Krisis », 2002, texte ${ }^{\circ}{ }^{\circ} 16$, pp. 443-462.

${ }^{2}$ Voir à cet égard le commentaire pénétrant que Marc Richir consacre à la circulation de l'affectivité dans la Phantasia chez Husserl dans son important ouvrage Phantasia, imagination, affectivité. Phénoménologie et anthropologie phénoménologique, Grenoble, éditions Jérôme Millon, coll. « Krisis », 2004, pp. 9-46. 
culpabilité liée à une faute morale qui aurait été commise. Si l'on peut difficilement faire l'économie de la dimension morale fondatrice de la honte, ce n'est pas exclusivement sur elle que nous porterons notre attention. En effet, ce qui va nous intéresser, c'est le rapport vécu au corps dans l'expérience que l'on peut faire de la honte. Toutefois, il semble que c'est précisément au niveau du corps, pour ne pas dire dans le corps, que se noue dans la honte le spectre d'une faute morale. Pour Eugen Fink, qui utilise le terme allemand Sham pour rendre compte à la fois de la pudeur et de la honte, l'association de la faute morale au corps résulte de l'héritage d'un idéalisme éthique issu de Platon (aidos). Selon cette conception éthique qui fonde la tradition occidentale, c'est la dimension spirituelle qui doit être privilégiée au détriment de la dimension corporelle. Il écrit que selon cette tradition,

Ce qui est éthique ne se présente que comme le rapport mondain que l'homme entretient avec l'esprit. C'est la raison pour laquelle demeure un résidu : la simple nature, également la nature en l'homme. Elle est un « reste de la terre », qu'il ne nous est pas facile de porter, tant que nous possédons encore un corps (Leib) ; l'âme ressent le corps comme un "cachot", comme une prison honteuse. À l'encontre du corps et de ses désirs animaux, la liberté de l'être spirituel doit être affirmée et défendue ${ }^{1}$.

Critiquant fermement le dualisme de l'âme et du corps et ses implications sur le plan éthique, Fink rejette vigoureusement la position qui consiste à faire de la pudeur une ré-action de l'esprit vis-à-vis du bestial. La pudeur, en tant qu'expérience fondamentalement antéprédicative, n'est pas un sursaut spirituel face à une nudité jugée honteuse ; la pudeur est partie intégrante de la nudité en tant qu'elle révèle le mystère de la nature en nous. Ce faisant, la pudeur est d'emblée, au cœur même de la nudité, préservation de ce mystère.

${ }^{1}$ Cf. E. Fink, «La nature en nous » (1951), trad. fr. N. Depraz, in Alter, nº 7, 1991, p. 273. On retrouve ici ce que décrit Foucault dans le corps utopique, à savoir cette utopie par laquelle l'âme cherche à effacer le corps : «Mais peut-être la plus obstinée, la plus puissante de ces utopies par lesquelles nous effaçons la triste topologie du corps, c'est le grand mythe de l'âme qui nous la fournit depuis le fond de l'histoire occidentale ». Cf. Michel Foucault, Le corps utopique, les hétérotopies, Fécamp, Nouvelles Éditions Lignes, 2009, p. 11. 


\section{Visibilité et reconnaissance : le regard d'autrui}

Cette dimension profondément mystérieuse du corps, décrite comme la « force énigmatique » de la nature en nous, Fink la célèbre en la rapprochant de la poésie. La poésie n'explique pas ; elle préserve le mystère tout en le dévoilant. Sur un tout autre mode, Sartre nous renvoie aussi à la dimension mystérieuse du corps, lorsqu'il s'interroge sur la vulnérabilité profonde de l'être-vu dans l'expérience du regard. Dans la troisième section de L'Être et le Néant, Sartre présente généralement le phénomène de la honte en le rapportant à une structure ontologique du pour-soi à part entière. Dans celleci, le pour-soi découvre un être qui est son être sans être pour-soi. Il écrit :

Je viens de faire un geste maladroit ou vulgaire : ce geste colle à moi, je ne le juge ni le blâme, je le vis simplement, je le réalise sur le mode du pour-soi. Mais voici tout à coup que je lève la tête : quelqu'un était là et m'a vu. Je réalise tout à coup la vulgarité de mon geste et $\mathrm{j}$ 'ai honte ${ }^{1}$.

L'expérience de la honte que décrit Sartre dans ce passage apparait indissociable de sa structure sociale, ou en tout cas intersubjective, dans la mesure où l'on ne peut avoir honte que devant quelqu'un. Le geste évoqué par Sartre a beau être déplacé, si l'individu qui l'accomplit est seul dans la pièce, s'il est assuré de pouvoir l'être, il ne vivra probablement pas l'effectuation de ce geste sur le mode de la honte. La honte est étroitement liée au phénomène de la visibilité ; elle révèle la structure ontologique de l'être pour autrui. Toutefois, il semble également que la dimension sociale de la honte dépasse le seul fait de la présence physique d'autrui.

La présence d'autrui signale en effet quelque chose de plus que Sartre thématise sous le terme de « reconnaissance ». Il écrit ceci : «La honte est, par nature, reconnaissance. Je reconnais que je suis comme autrui me voit $»^{2}$. Contrairement à ce que pourrait laisser entendre ce terme, la reconnaissance qui a lieu ne procède pas d'une opération réflexive. Cette reconnaissance se caractérise par son immédiateté ; elle apparaît comme une épreuve aussi surprenante qu'évidente. Cette remarque est importante car elle indique que le phénomène de la honte est intrinsèquement lié à l'intimité, à la corporéité, sans détour préalable par le spirituel. Dans cette perspective, si l'idée d'une reconnaissance immédiate, d'une reconnaissance sur-le-champ peut sembler paradoxale, l'usage du terme reconnaissance permet de

${ }^{1}$ J.-P. Sartre, L'Être et le Néant. Essai d'ontologie phénoménologique (1943), Paris, Gallimard, coll. « Tel», 2013, p. 259-260.

${ }^{2}$ Ibid., p. 260. 
manifester que l'expérience de la honte s'accompagne d'une certaine violence, ne serait-ce que par son caractère brutal. Sartre insiste bien sur cet aspect lorsqu'il indique que si j'apparais, dans la honte, comme objet sous le regard d'autrui, cet objet

n'est pas une vaine image dans l'esprit d'un autre. Cette image en effet serait entièrement imputable à autrui et ne saurait me «toucher». Je pourrais ressentir de l'agacement, de la colère en face d'elle, comme devant un mauvais portrait de moi, qui me prête une laideur ou une bassesse d'expression que je n'ai pas, mais je ne saurais être atteint jusqu'aux moelles ${ }^{1}$.

En d'autres termes, l'expérience de la honte est la découverte de mon intimité exposée et révélée dans et par la présence d'autrui. Et c'est dans mon corps que j'éprouve cette intimité dévoilée dans le mouvement même où mon corps pour moi devient corps pour autrui.

En ce sens, si la honte est structurellement sociale, il nous semble que c'est à deux niveaux distincts, qui s'entrecroisent constamment, que l'on peut analyser le phénomène de reconnaissance qui a lieu dans la honte.

D'une part, la reconnaissance telle qu'elle se déploie dans la honte renvoie à la découverte de la vulnérabilité essentielle de mon être en tant que mon corps est toujours potentiellement un corps pour autrui. Plus précisément, ce corps que je suis, parce que je ne peux que le vivre sur le mode du pour-soi, m'échappe ; il est «le point de vue sur lequel je ne puis plus prendre de point de vue $»^{2}$. Si je suis atteint jusqu'aux moelles dans la honte, c'est qu'autrui me révèle à moi-même un point de vue qui m'échappe absolument. Qui plus est, c'est la découverte d'un point de vue que je ne peux seulement vivre et ressentir qu'à l'occasion du regard d'autrui. La vulnérabilité foncière de mon être visible pour autrui se donne avant tout comme l'expérience d'une nudité originaire. Pour reprendre le mot d'Agamben, je me vis comme tout à coup « exproprié ${ }^{3}{ }^{3}$ 'un point de vue qui me manque radicalement, mais qui se donne à vivre - et c'est loin d'être un détail - de «l'intérieur ». La reconnaissance qui a lieu est celle de l'épreuve douloureuse que seul autrui constitue « le médiateur indispensable entre moi et moi-même ${ }^{4}$. Aussi, avant d'être l'image de quelque chose, avant que de

\footnotetext{
${ }^{1}$ Ibidem

${ }^{2}$ Ibid., p. 369.

${ }^{3}$ G. Agamben, Ce qui reste d'Auschwitz. L'archive et le témoin. Homo Sacer III (1998), trad. fr. P. Alferi, Paris, Éditions Payot \& Rivages, coll. Rivage Poche/Petite Bibliothèque, 2003, p. 114.

${ }^{4}$ J.-P. Sartre, L'Être et le Néant, op. cit., p. 260.
} 
porter la signification d'un geste vulgaire, la honte que je ressens est avant tout honte d'avoir été vu. Comme l'analyse parfaitement Sartre dans le cadre de l'embarras, je peux chercher tant bien que mal à maîtriser mon corps pour autrui ; cependant, je ne peux jamais l'atteindre : « C'est au contraire parce qu'il n'est jamais là, parce qu'il demeure insaisissable, qu'il peut être gênant $\gg{ }^{1}$. À notre sens, cette dimension mystérieuse du corps peut être rapprochée du corps utopique que Foucault décrit sur un mode phénoménologique dans une conférence du même nom en 1966. Le corps utopique rend compte en effet de ce paradoxe saisi par Sartre dans L'Etre et le Néant : c'est justement parce que le corps est lieu absolu — je ne peux pas m'en défaire, comme le dit Foucault, je ne peux m'en aller et le laisser derrière moi qu'il m'échappe de toutes parts. Seules l'expérience du corps pour autrui (Sartre) et celle du miroir (pour Foucault) semblent pouvoir le localiser, le spatialiser, sans pour autant jamais épuiser les pans entiers de visibilité et d'invisibilité du corps.

D'autre part, si l'expérience de la honte est immédiate, si elle est intimement vécue "sans aucune préparation discursive »" la structure sociale de la honte suggère de faire état d'un autre aspect qui, cette fois, fait appel au registre de l'image, de la représentation, ou encore de la signification. Dans ce cadre, la question de la nudité devient davantage une métaphore. Comme le montrent les analyses de Levinas dans De l'évasion, "la honte apparaît chaque fois que nous n'arrivons pas à faire oublier notre nudité. Elle a rapport avec tout ce que l'on voudrait cacher et que l'on ne peut pas enfouir $»^{3}$. Au niveau social, Levinas prend dans son ouvrage entre autres exemples celui de la pauvreté. Les guenilles du pauvre instituent à elles seules sa nudité et même lui assurent cette nudité en tant que le pauvre témoigne "d'une existence incapable de se cacher ${ }^{4}$. L'existence incapable de se cacher est de nature profondément sociale dans la mesure où elle se caractérise par un excès de visibilité qui, paradoxalement, condamne un individu à demeurer invisible. L'impossibilité de se cacher, c'est, d'une manière ou d'une autre, subir un excès de visibilité qui rend radicalement opaque toute autre perception et, de ce fait même, brise le processus de reconnaissance ${ }^{5}$.

\footnotetext{
${ }^{1}$ Ibid., p. 394.

${ }^{2}$ Ibid., p. 260.

${ }^{3}$ E. Levinas, De l'évasion (1935), Paris, Fata Morgana, 1982, p. 112.

${ }^{4}$ Ibidem

${ }^{5}$ Cf., à propos de la problématique de la visibilité, A. Honneth, "Visibilité et invisibilité. Sur l'épistémologie de la "reconnaissance" », trad. fr. F. Gollain \& C.
} 
Afin de mieux comprendre les liens entre la structure sociale de la honte et la reconnaissance, on peut évoquer également le roman autobiographique d'Annie Ernaux intitulé La Honte. Dans ce roman, Annie Ernaux décrit sa jeunesse au début des années cinquante dans une petite ville provinciale de Normandie. Elle y raconte de quelle manière elle est « entrée dans la honte $»^{1}$ lorsque, au mois de juin 1952, son père, excédé par une énième dispute conjugale, tente d'étrangler sa mère sous ses yeux. Ses parents tiennent une sorte de café-épicerie où se rencontrent deux mondes qui coupent en deux aussi bien géographiquement que socialement la ville d'Yvetot. Elle décrit à ce propos de manière exemplaire la répartition sociale qui met, d'un côté, ceux qui sont capables de se cacher et, de l'autre, « ceux dont on sait ce qu'ils touchent comme allocations, ce qu'ils mangent et boivent, à quelle heure ils se couchent $»^{2}$.

La jeune fille qu'elle était alors semble tiraillée entre ces deux mondes. Elle est à la fois la jeune fille dont la mère, pieuse, l'inscrit à l'école privée - symbole à elle seule d'une ambition sociale prometteuse - et à la fois la jeune fille qui voit ses parents sombrer peu à peu et «perdre» leur dignité suite à la scène de l'étranglement. Dans le passage suivant, Annie Ernaux évoque la honte que lui a procuré un jour la vision de sa mère, alors qu'elle rentrait d'une fête de la jeunesse des écoles chrétiennes. Elle écrit :

J'ai frappé contre le volet de la porte de l'épicerie. Après un temps assez long, l'électricité s'est allumée dans le magasin, ma mère est apparue dans la lumière de la porte, hirsute, muette de sommeil, dans une chemise de nuit froissée et tachée (on s'essuyait avec, après avoir uriné). Mlle L. et les élèves, deux ou trois, se sont arrêtées de parler. Ma mère a bredouillé un bonsoir auquel personne n'a répondu. Je me suis engouffrée dans l'épicerie pour faire cesser la scène. Je venais de voir pour la première fois ma mère avec le regard de l'école privée ${ }^{3}$.

Analysant le souvenir de cette expérience honteuse, l'auteur ajoute : «Comme si à travers l'exposition du corps sans gaine, relâché, et de la chemise douteuse de ma mère, c'est notre vraie nature et notre façon de vivre qui étaient révélées $\gg{ }^{4}$.

Lazzeri, in Revue du Mauss, 2004/1, n²3, p. 137-151 ; G. le Blanc, L'invisibilité sociale, Paris, P.U.F, coll. « Pratiques théoriques », 2009.

${ }^{1}$ A. Ernaux, La Honte, Paris, Gallimard, coll. Folio, 1997, p. 116.

2 Ibid., pp. 50-51.

${ }^{3}$ Ibid., p. 118.

${ }^{4}$ Ibidem 
Dans ce dernier extrait, Annie Ernaux nous permet d'explorer de quelle manière on a non seulement toujours honte devant quelqu'un, mais comment, également, l'expérience de la honte fige, par-delà certaines représentations, un individu dans une image. Comme le note Sartre luimême, dans l'expérience de la honte, ce sont les possibilités d'un individu qui se retrouvent figées. Sartre écrit en effet à ce propos : " Le corps-pourl'autre c'est le corps-pour-nous, mais insaisissable et aliéné »" ${ }^{1}$. Si la honte colle à la peau, c'est parce que le regard d'autrui semble fixer, comme pour l'éternité, la totalité de mon être dans l'une de ses possibilités ; de telle sorte que je ne puis y échapper, de telle sorte que je ne puis à jamais être rien d'autre que cette image. Pour le dire dans les termes de Levinas, dans la honte éprouvée par Annie Ernaux face à la vision de sa mère, ce qui se dévoile, c'est l'impossibilité de " couvrir la nudité de sa personne physique par sa personne morale $»^{2}$. Par ailleurs, l'expérience de honte vécue par Annie Ernaux montre que l'épreuve de la honte n'est pas inhérente à l'individu effectuant un geste vulgaire ou affichant un comportement déplacé. Il y dans la honte une dimension virale qui semble se propager dans le cadre même des limites de la visibilité.

Mais si, comme l'énonce Sartre, autrui assure une fonction essentielle en nous donnant à nous voir tels que nous sommes, autrui ne constitue pas pour autant un miroir ${ }^{3}$. Il faut approfondir l'analyse de la structure sociale de la honte et de la manière dont, à travers la fixation dans l'une ou l'autre possibilité, l'individu qui fait l'expérience de la honte se trouve douloureusement malmené dans le mouvement même où il se reconnaît à travers le regard d'autrui. Comment cette reconnaissance a-t-elle lieu exactement? D'où procède-t-elle? Dans L'Être et le Néant, Sartre insiste bien sur le fait que si le regard d'autrui me chosifie, si autrui se révèle à moi comme sujet dans le sens où je deviens, pour lui, un objet sous son regard, cela ne signifie pas que les significations dans lesquelles je me retrouve figé seraient de son propre fait. Pas davantage autrui ne me révélerait des qualités intrinsèques de mon être, qualités qui seraient jusque-là demeurées à l'état latent. Sartre montre bien, à propos de ces qualifications nouvelles, qu'il est impossible qu'autrui ne les tire jamais de mon être pour-moi. Comment le pourrait-il

\footnotetext{
${ }^{1}$ J.-P. Sartre, L'Être et le Néant, op. cit., p. 394.

${ }^{2}$ E. Levinas, De l'évasion, op.cit., p. 112.

3 Autrui n'est pas un miroir; il fait plutôt effraction dans mon intimité et me découvre exproprié, au sein de mon intimité, d'une manière d'être visible de mon corps que je ne contrôle pas.
} 
seulement, étant donné que l'expérience de la honte se situe d'emblée sur le plan préréflexif?

Par conséquent, il faut affirmer avec Sartre que ce n'est qu'à l'occasion du regard d'autrui que certaines représentations peuvent être fixées et figer la totalité de mon être. Pour le dire avec les mots de L'Être et le Néant, «la notion même de vulgarité implique d'ailleurs une relation intermonadique. On n'est pas vulgaire tout seul ${ }^{1}$. En d'autres termes, ce que Sartre nous permet de penser ici, c'est que ce serait reconduire la honte à la seule faute morale que d'en faire une spécificité intrinsèque de tel ou tel individu. Attribuer le trait honteux d'une expérience à un individu comme s'il s'agissait d'une caractéristique propre de sa personne, c'est nier, paradoxalement, tout ce que la honte peut avoir de social. C'est reporter le tout de l'expérience honteuse du côté de la reconnaissance, en instaurant alors une responsabilisation individuelle écrasante. C'est, finalement, faire comme si le regard d'autrui n'était qu'un simple miroir, comme si les qualités nouvellement posées émanaient seulement et directement du pour-soi.

\section{Une « subjectivité coupable » : être rivé à soi}

En ce sens, il nous semble que la mise en perspective des analyses que l'on peut faire de la honte chez Sartre avec les propos d'Alain Brossat concernant la culpabilisation intense des chômeurs peut être éclairante. Sur le plan politique, Alain Brossat indique de quelle manière il s'agit, selon lui en France, de produire une « subjectivité coupable $»^{2}$. Il s'agit de faire en sorte que les individus se sentent coupables - et dès lors responsables - d'être chômeurs, d'être sans emploi, et, bien sûr, de refuser un travail qui ne convient pas à leurs qualifications, etc. Plus spécifiquement, il nous semble que l'analyse sartrienne de la honte, bien qu'elle ne le mette pas explicitement en évidence, invite à réfuter fermement l'idée — si commune - selon laquelle l'individu qui expérimente la honte se situe dans un rapport exclusivement personnel à l'égard de la honte ressentie. Si la honte colle effectivement à la peau, mais si, également, on n'est pas vulgaire tout seul, c'est que l'individu qui se comporte de manière vulgaire ne chute pas seul dans la honte. En m'exposant sa vulgarité comme l'une des possibilités de

\footnotetext{
${ }_{1}^{1}$ J.-P. Sartre, L'Être et le Néant, op. cit., p. 260.

${ }^{2}$ M. Vander Elst, Entretien avec Alain Brossat. Discussion autour de Autochtone imaginaire, étranger imaginé, Bruxelles, février 2014.

Cf. Hhttps://www.academia.edu/12479728/Entretien_avec_Alain_Brossat
}

\section{1}


son être, l'individu m'entraîne avec lui dans la honte, simplement parce que ce que je reconnais tout à coup, c'est que la vulgarité constitue une possibilité fondamentale de toute réalité-humaine - y compris de mon être.

Il n'est pas certain, comme nous venons de l'indiquer, que Sartre emprunte effectivement cette voie pour rendre compte du caractère structurellement social de la honte. En effet, il faut garder à l'esprit le fait que l'expérience de la honte est, sur le plan sartrien, une expérience marquée par une asymétrie : je ne peux me vivre comme objet sous le regard d'autrui que si celui-ci se donne à moi comme sujet, sujet dont je suis précisément l'objet. En conséquence, je ne peux être chosifié, réduit à l'en-soi par le regard d'autrui que si ce dernier m'apparaît comme sujet et, comme tel, libre. Il m'est impossible de me vivre comme aliéné par autrui si ce dernier ne fait pas l'exercice de sa liberté. Il n'y a d'objectivation que là où il y a liberté. On pourrait donc être très tenté de réduire le regard d'autrui à une posture extérieure s'épuisant dans sa fonction de catalyseur. Et pourtant, bien que cette lecture soit tenable jusqu'à un certain point, il faudrait admettre, d'une part, que la dimension honteuse d'une situation incombe radicalement et absolument à l'individu qui l'expérimente et ce, comme quelque chose qui lui appartient en propre. D'autre part, une telle lecture supposerait alors de rétrécir considérablement le cadre moral dans lequel surgit le phénomène de la honte en identifiant celle-ci à la culpabilité. Effectivement, en identifiant la honte à un sentiment de culpabilité personnelle, on risque de replacer la singularité de la honte au niveau spirituel, en oblitérant tout ce que la honte peut avoir de corporel. Ce serait oublier, comme l'écrit Sartre, que les significations qu'autrui m'applique " renvoient à la fois à un témoin susceptible de les comprendre et à la totalité de ma réalité-humaine $»^{1}$. Pour le dire autrement, avant d'analyser plus avant cette figure du témoin, notre hypothèse est qu'il $\mathrm{y} a$, dans le phénomène de la honte, un effet de contamination et de contagion qui fait sombrer dans la honte les deux protagonistes en présence. L'un ne saurait, au détriment de l'autre, se targuer d'en sortir indemne.

Quel rapport peut-on alors établir entre l'analyse sartrienne de la honte et la dénonciation, par Alain Brossat, de la production d'une «subjectivité coupable»? À notre sens, les propos d'Alain Brossat quant au processus de culpabilisation à outrance des chômeurs expriment parfaitement tout ce que la honte a de social et ce, jusque dans les situations où elle semble n'être que personnelle. Lorsqu'un individu est confronté à une situation dans laquelle autrui fait l'épreuve de la honte, il s'agit toujours, d'une manière ou d'une

${ }^{1}$ J.-P. Sartre, L'Être et le Néant, op. cit., p. 260. 
autre, pour cet individu, de se désolidariser au plus vite de ce qui le touche déjà, de ce qui l'atteint déjà. Plus explicitement, lorsque j'assiste à l'épreuve qu'autrui fait de la honte, il s'agit toujours déjà de m'extirper d'une situation dans laquelle je suis d'emblée taché par ce qui m'éclabousse. Faire porter à autrui la responsabilité exclusive de l'expérience honteuse, lui conférer une propriété personnelle, c'est tenter de fuir la solidarité intime et mystérieuse qui me lie à autrui ${ }^{1}$. Autrement dit, dans l'exposition de la vulnérabilité d'autrui, c'est aussi ma vulnérabilité qui se révèle à moi.

Par conséquent, sur le plan de la culpabilisation des chômeurs, il s'agit, comme le dit Alain Brossat, d'une

mise en condition - car il faut que ces gens-là soient installés dans une subjectivité coupable. Qu'ils se sentent coupables de ce qu'ils n'ont pas fait. C'est le jeu du gouvernement moderne : faire en sorte que les gens se sentent coupables d'une faute qui est mal établie ou imaginaire. Rouler avec une voiture qui fonctionne au diesel, par exemple. Créer cette culpabilité passe par ce type de dispositif, assez sophistiqué et très pervers ${ }^{2}$.

Cette "mise en condition » consiste en un modelage des subjectivités par lequel on tente d'incorporer chez le sujet la responsabilité totale de sa situation. Il ne s'agit pas seulement de mettre en place et d'instituer ce que Alain Ehrenberg nomme une «norme d'autonomie $»^{3}$. Dans le sillage des

\footnotetext{
${ }^{1}$ Voir à cet égard l'excellente étude de terrain réalisée par Pascale Jamoulle sur les implications des politiques d'urbanisation dans les cités ouvrières des années 1960 dans Des Hommes sur le fil. La construction de l'identité masculine en milieux précaires, Paris, La Découverte, 2008. Mettant en évidence les conséquences de la désindustrialisation sur ces quartiers fortement précarisés par le chômage de masse et reconvertis en logements sociaux, l'auteur montre comment leur stigmatisation renforce un profond sentiment de honte chez leurs habitants. Comme l'écrit Pascale Jamoulle : «L'habitat est comme une seconde peau, il est le support spatialisé des constructions identitaires » (p. 13). Loin de déboucher sur une solidarité renforçant la cohésion sociale au sein de la cité, la ségrégation et le sentiment permanent de honte qui en découle isolent davantage les individus. "Le stigmate d'allocataire social isole les résidents, produit des sentiments de honte et des rapports sociaux de défiance mutuelle. Ségrégation et stigmatisation spatiales semblent fonctionner comme des matrices qui engendrent, à l'intérieur des groupes disqualifiés, de nouvelles formes de classement et d'exclusion, toujours plus violentes » (p. 26).

${ }^{2}$ M. Vander Elst, Entretien avec Alain Brossat. Discussion autour de Autochtone imaginaire, étranger imaginé, op.cit.

${ }^{3}$ Cf. A. Ehrenberg, L. Mingasson \& A. Vulbeau, « L'autonomie, nouvelle règle sociale. Entretien avec Alain Ehrenberg », in Informations sociales, 2005/6, $\mathrm{n}^{\circ} 126$,
} 
analyses de Foucault, il s'agit de montrer que, dans une situation de crise qui voit le plein-emploi classé au rang d'époque révolue, cette pratique d'incorporation d'une responsabilité sans limite chez les sujets révèle une dynamique perverse. En effet, comme le dit Alain Brossat, «plus on va accroître les possibilités de montrer qu'en réalité le demandeur d'emploi ne se conforme pas à ce qui est exigé de lui, mieux on va pouvoir le radier ${ }^{1}$. Autrement dit, l'enjeu essentiel d'une culpabilisation et d'une responsabilisation à outrance des chômeurs est de créer les conditions de ce que l'on cherche exactement à démontrer, à savoir : le manque de bonne volonté du chômeur, son manque d'adaptabilité, mais aussi de flexibilité. Ce faisant, par le fait d'une hyper-responsabilisation, on crée chez le chômeur un sentiment de culpabilité puissant qui le pousse à se vivre comme la cause essentielle de son échec. De plus, la perversité d'une telle logique implique une isolation importante qui empêche les chômeurs, paradoxalement, d'avoir accès aux réseaux et aux structures qui leur permettraient de sortir de l'impasse dans laquelle ils sont pris. Ce qui conduit à comprendre ceci : la précarité n'est pas «simplement» quelque chose que l'on comptabilise; elle est, de part en part, structurelle.

Pour en revenir à Sartre, il nous semble que le recours à la figure du témoin pour caractériser le regard par lequel autrui me chosifie peut donc apporter un élément important pour la compréhension de la honte en tant que phénomène social. On peut se demander si ce témoin renvoie à autre chose que la totalité de la réalité-humaine. Sartre permet par cette évocation de la figure du témoin d'évoquer ce que, très justement, Derrida appelle une honte «honteuse d'elle-même $»^{2}$. Derrida analyse cette honte honteuse d'ellemême dans le cadre d'une situation spécifique, celle d'être « à poil devant un chat $»^{3}$. Bien que la situation décrite et imaginée par Derrida puisse sembler humoristique, celui-ci met en lumière que la honte honteuse d'elle-même désigne non seulement l'épreuve de la honte en tant que telle, mais implique

pp. 112-115. Dans cet entretien, quelques années après la parution de La fatigue d'être soi (1998), Alain Ehrenberg montre que, loin d'en appeler à la dimension exclusivement individuelle de réalisation de soi, l'autonomie constitue au contraire une règle sociale. Il écrit : «L'individualisme est en général assimilé à l'idée que la règle sociale s'affaiblit, alors qu'il est le corrélat d'une transformation de la règle, à savoir le progressif englobement des références disciplinaires dans celle de l'autonomie » (p. 113).

${ }^{1}$ M. Vander Elst, Entretien avec Alain Brossat. Discussion autour de Autochtone imaginaire, étranger imaginé, op.cit.

2 J. Derrida, L'animal que donc je suis, Paris, Galilée, 2006, p. 27.

${ }^{3}$ Ibid., p. 18. 
également une intensification de l'épreuve de la honte comme honte d'avoir honte. Il y aurait donc, dans la honte, un effet de contamination qui s'accompagne d'une intensification par laquelle la honte ne cesse de s'alimenter. Selon nous, cette situation d'intensification n'est possible que parce qu'autrui, lorsqu'il me regarde, est davantage qu'un simple spectateur. La honte d'avoir honte suggère qu'autrui, dans le mouvement même au cours duquel il m'applique de nouvelles qualités, éprouve lui aussi de la honte.

Par l'évocation d'une honte honteuse d'elle-même, la figure sartrienne du témoin nous paraît entrer en résonance avec la question posée par Derrida dans L'animal que donc je suis, celle du « qui ? ». Derrida amène à se poser cette question en se demandant devant qui, finalement, a-t-on honte lorsqu'on se trouve nu devant un chat. Très subtilement, Derrida pose le problème en ces termes :

Mais honteuse d'elle-même, cette honte est plus intense, je dois aussitôt le souligner, quand je ne suis pas seul avec la chatte dans la pièce. Alors je ne suis plus sûr de savoir devant qui je suis ainsi transi de peur. Est-on d'ailleurs jamais seul avec un chat? Et avec quiconque ? Ce chat, est-ce un tiers ? ${ }^{1}$

Selon nous, cette question du tiers est également posée par Sartre lorsqu'il introduit le terme de témoin. Si nous avons déjà longuement insisté jusqu'ici sur la manière dont le phénomène de la honte, étroitement lié à la visibilité, engage une dialectique de la reconnaissance, c'est cette dernière que la question du tiers suggère de préciser.

De quoi en effet le regard d'autrui est-il le témoin ? C'est précisément à cette question que répond Derrida. Le témoin sartrien se découvre à luimême et à l'autre comme témoin du tiers. Qu'est-ce que cela signifie ? Cela signifie que lorsque je me découvre honteux à autrui, bien que cela ne puisse se produire qu'à l'occasion de sa présence, ce dernier apparaît porteur d'un tiers auquel pourtant on ne saurait l'identifier. Plus concrètement, la position du témoin sartrien comme catalyseur est révélation d'un tiers, sans pouvoir y être réduit pour autant. Qu'est-ce que ce tiers? Notre hypothèse est que ce tiers renvoie à l'ensemble des normes, des codes. Plus spécifiquement, ce tiers constitue, pour une société donnée, l'ensemble de ses convenances, de ses normes et de ses valeurs sociales, morales, religieuses et culturelles. Ce tiers se révèle, dans l'expérience honteuse, dans la relation que la honte fait émerger entre deux individus. Ce tiers surgit pour l'un et l'autre des deux individus en présence, sans qu'on ne puisse jamais le définir comme une

${ }^{1}$ Ibid., p. 27. 
caractéristique personnelle inhérente à l'un ou l'autre. Toutefois, on pourrait objecter que faire l'hypothèse d'un tiers, c'est prendre le risque d'enfermer le phénomène de la honte dans un cadre moral restrictif. La honte ne serait alors rien d'autre que le fait de commettre une faute. Au contraire, nous souhaiterions souligner que faire l'hypothèse du tiers, ce n'est pas réintroduire au sein de la conscience une morale précédant toute situation et toute action. Il s'agit plutôt d'affirmer qu'il n'y a émergence d'une problématique morale qu'au sein d'une rencontre entre deux individus ${ }^{1}$.

Sur ce point, on peut faire également une seconde objection. L'épreuve de la honte, que nous avons décrite comme fondamentalement sociale, peutelle être vécue hors de tout contexte social? Si honte et visibilité sont étroitement liées, est-il possible d'envisager une expérience privée de la honte? On pourrait se demander en effet, comme Derrida, ce que signifie être «seul» et si on peut jamais l'être. Autrement dit, est-ce qu'être seul n'est jamais qu'une autre manière d'être avec les autres? Dans L'Etre et le Néant, Sartre s'interroge sur la possibilité d'une "honte devant personne » ${ }^{2}$ et définit alors l'absence comme une modalité spécifique d'être-là de la réalité humaine :

Me voilà courbé sur le trou de la serrure ; tout à coup j'entends des pas. Je suis parcouru par un frisson de honte : quelqu'un m'a vu. Je me redresse, je parcours des yeux le corridor désert : c'était une fausse alerte. Je respire. (...) Loin qu'autrui ait disparu avec ma première alerte, il est partout présent, en dessous de moi, au-dessus de moi, dans les chambres voisines et je continue à sentir profondément mon être-pour-autrui ; il se peut même que ma honte ne disparaisse pas $(\ldots)^{3}$.

\footnotetext{
${ }^{1}$ La question que nous nous posons est la suivante : ce tiers ne préexiste pas à la relation à autrui et pourtant, ce que met en évidence Sartre dans cette troisième section de L'Être et le Néant, c'est la structure autrui comme présence originelle. Autrui est toujours déjà là, comme structure. Toutefois, l'expérience de la honte nous montre que la problématique morale surgit toujours en relation avec autrui, qu'il soit présent ou absent, c'est-à-dire qu'il apparaisse sous cet autre mode de présence qu'est l'absence. Si l'on peut dire qu'autrui est toujours là, même absent, même dans le bruissement des feuilles qui me surprend, cela signifie que mon expérience du monde est toujours indissociable de valeurs. Ce qui ne signifie pas restreindre ces valeurs sur un plan moral aussi étroit que celui de la faute, mais ce qui signifie qu'il n'y a jamais d'expérience neutre du monde, de mon environnement. Elle apparaît toujours déjà immergée dans des valeurs.

${ }^{2}$ J.-P. Sartre, L'Être et le Néant, op. cit., p. 315.

${ }^{3}$ Ibid., p. 316.
} 
Sartre montre dans ce passage que je suis toujours susceptible d'être regardé et cette potentialité permanente fait de mon être-pour-autrui non un accident dans mon existence, mais un « fait constant de ma réalité-humaine » ${ }^{1}$. Aussi, loin d'être une « honte fausse $»^{2}$, la situation décrite par Sartre suggère que je suis toujours un être-pour-autrui sans pour autant être d'emblée et constamment regardé. Toutefois, la honte désigne une expérience dans laquelle, plus que jamais, j'ai conscience de mon être-pour-autrui et où se révèle mon êtreobjet pour l'autre. Sartre va même plus loin, considérant qu'autrui constitue une " présence originelle ${ }^{3}$. C'est cette présence originelle d'autrui qui organise mon existence comme un espace dans lequel je me trouve toujours, quoi qu'il arrive, à une distance relativement proche ou lointaine d'autruiobjet. Comme l'indique Sartre, ce qui est alors seulement et toujours probable, c'est, d'une part, la proximité relative d'autrui et, d'autre part, que je sois pour autrui tel ou tel objet.

Dès lors, s'il apparaît impossible de rendre compte de l'expérience de la honte en dehors de sa dimension sociale, il demeure un aspect de la problématique de la honte que nous n'avons fait qu'évoquer jusqu'à présent sans l'approfondir. Effectivement, nous nous sommes attachée à analyser le processus dialectique de reconnaissance en jeu dans le phénomène de la honte. Toutefois, comme le fait remarquer Levinas, on oublie régulièrement la dimension proprement personnelle de la honte en privilégiant de mettre l'accent sur l'aspect social. Or, comme l'écrit Levinas :

Si la honte est là, c'est que l'on ne peut pas cacher ce que l'on voudrait cacher. La nécessité de fuir pour se cacher est mise en échec par l'impossibilité de fuir. Ce qui apparaît dans la honte, c'est donc précisément le fait d'être rivé à soi-même, l'impossibilité de fuir pour se cacher à soi-même, la présence irrémissible du moi à soi-même ${ }^{4}$.

Pour Levinas, dans la honte, je fuis la situation précisément parce que je reconnais que c'est bien de moi dont il s'agit. Sur ce point, les analyses de Sartre et de Levinas sont assez proches, bien que ce dernier n'identifie pas explicitement la fonction jouée par autrui lorsqu'il me regarde. Levinas insiste davantage sur cette dialectique entre moi et moi-même en la décrivant pour elle-même. Il montre que le mouvement de fuite procède d'une reconnaissance qui a bien eu lieu mais qu'il est impossible d'assumer et de

${ }^{1}$ Ibid., p. 319.

${ }^{2}$ Ibid., p. 315.

${ }^{3}$ Ibid., p. 319.

${ }^{4}$ E. Levinas, De l'évasion, op.cit., p. 113. 
s'approprier. Dans la honte, je reconnais que c'est manifestement de moi dont il est question et pourtant, il m'est impossible de m'identifier à ce que j'ai pu faire. Dans une telle situation, je suis mû par le besoin de fuir la répugnance que je ressens à l'égard d'un acte que je sais être de mon fait. Cette situation de détresse en suscite une autre, celle de l'impossibilité de me fuir. Fuir le lieu de ma situation ne servirait à rien : il apparait qu'il n'existe aucune évasion possible devant soi-même. Levinas, ce faisant, semble isoler le sujet dans une responsabilité qu'il subit sans pouvoir la faire sienne. Je ne peux reconnaître pleinement ce que j'ai fait sans, du même coup, m'anéantir.

C'est en ce sens que, reprenant les analyses de Levinas dans Ce qui reste d'Auschwitz, Agamben prolonge celles-ci en apportant un nouvel éclairage sur le processus dialectique à l'œuvre dans la honte. Selon Agamben, l'analyse levinassienne du sujet honteux peut être interprétée comme une dialectique de subjectivation/désubjectivation. Pour Agamben, le sujet honteux décrit par Levinas est un sujet « livré à une altérité inassumable »" Selon le philosophe italien, c'est précisément la honte qui permet de révéler la dialectique qui a lieu dans l'expérience que fait le sujet. Être sujet, c'est constamment être pris au sein de ce processus de subjectivation/désubjectivation. Tout mouvement de subjectivation, pour être tel, s'accompagne d'un mouvement de désubjectivation. Agamben écrit à ce propos : «Dans la honte, le sujet a donc pour seul contenu sa propre désubjectivation : témoin de sa propre débâcle, de sa propre perte comme sujet (...) $\rangle^{2}$.

Ce détour par Agamben a le mérite, selon nous, de conceptualiser la détresse d'un sujet reconnaissant une responsabilité qu'il ne peut pourtant incarner. En effet, l'expression d' « altérité inassumable " forgée par Agamben rend compte de l'incompréhension que peut ressentir tout individu face à son action. L'action, à travers l'impossible anticipation de tous ses résultats, révèle au sujet une expérience de l'étrangeté. En plaçant l'épreuve de l'étrangeté au sein même du rapport à soi, Agamben invite selon nous à décrire l'expérience de la honte comme l'émergence d'une situation qui nous échappe. L'expression d' «altérité inassumable» permet d'atteindre ces caslimites - et pourtant si courants - dans lesquels je ne peux plus être moimême sans du même coup m'anéantir. Ce concept permet de relire les analyses de Levinas en insistant sur l'incompréhension qui m'assaille. Cette altérité, je ne peux l'assumer et pourtant elle demeure bien la mienne ; c'est bien de moi dont il est question: c'est bien moi qui ai agi de telle et telle façon et, pourtant, je ne peux me résoudre à m'y reconnaître. Chez Levinas,

${ }^{1}$ G. Agamben, Ce qui reste d'Auschwitz, op.cit., p. 114.

${ }^{2}$ Ibidem 
mais chez Agamben également, toute possibilité d'évasion qui consisterait à se préserver de cette altérité inassumable est exclue.

\section{Fuir par le corps : une tentative d'évasion?}

À cet égard, on peut trouver, dans le roman d'Annie Ernaux, le récit d'une tentative d'évasion qui semble amener la narratrice à se couper d'elle-même au fil des années. Plus exactement, cette coupure s'exerce au niveau du corps lui-même. Au début du roman, la narratrice décrit une photo prise le jour de sa communion. Alors qu'elle décrit celle-ci, elle fait part de son malaise à la vue de son corps. Elle écrit : "Impression qu'il n'y a pas de corps sous cet habit de petite bonne sœur parce que je ne peux pas l'imaginer, encore moins le ressentir comme je ressens le mien maintenant. Étonnement de penser que c'est pourtant le même aujourd'hui $\gg{ }^{1}$. Dans ce passage du livre, on découvre de quelle manière, pour survivre à la honte, pour tenter de lui échapper, la narratrice se désolidarise de son corps. Si la narratrice ne peut échapper à sa situation, si elle ne peut la fuir, la seule possibilité d'évasion se situe là, au sein même de l'intimité du corps et de la honte. La seule chance d'évasion, c'est de savoir qu'il s'agit bien de son corps sans pouvoir pour autant l'incarner. S'évader, c'est alors abandonner ce corps au passé et le vivre sans pouvoir se l'approprier. Le corps de la honte est alors un corps en suspens ; c'est un corps-pour-autrui qui n'en finit plus d'être absent.

Jusqu'à présent, nous nous sommes efforcée d'explorer en quoi la honte, en tant que phénomène social, ne peut être réduite à une faute morale qui révélerait la vraie nature ou le moi profond d'un individu, comme si cette honte le définissait de part en part. C'est en ce sens que nous avons convoqué les analyses de Sartre, celles d'Alain Brossat sur le chômage comme processus de production de subjectivités coupables et les descriptions du roman d'Annie Ernaux. Comme on l'a vu, la reconnaissance qui a lieu à l'occasion du regard d'autrui dans L'Être et le Néant est aliénante, dans le sens où le regard réifie l'autre à son corps pour autrui. Exproprié d'un point de vue dont il ne peut disposer sur son propre corps, l'individu qui fait l'épreuve de la honte se trouve figé dans un ensemble de significations qui, comme par magie, brisent ses possibilités. Toutefois, et c'est ce que nous souhaiterions approfondir dans la dernière partie de notre parcours, les analyses de Foucault dans $«$ Le corps utopique $»^{2}$ peuvent peut-être permettre de nuancer

${ }^{1}$ A. Ernaux, La Honte, op.cit., pp. 23-24.

${ }^{2} \mathrm{M}$. Foucault, Le corps utopique, les hétérotopies, op. cit. 
et d'envisager autrement cet enfer aliénant perpétuel auquel Sartre semble livrer le corps pour autrui du pour-soi. De cette façon, nous tenterons de creuser davantage les rapports entre corps et imagination et la façon dont le corps peut déployer des tentatives d'évasion.

«Le corps utopique » est un texte que Foucault a prononcé, avec celui sur les " hétérotopies », en décembre 1966 sur France Culture. Présentés comme des «essais littéraires», ils constituent, comme nous l'apprend Philippe Sabot, un document singulier au sein de l'œuvre de Foucault. Contemporain de la parution des Mots et les choses, il est frappant de constater que "Le corps utopique » constitue une véritable méditation phénoménologique sur le corps. Toutefois, celui-ci est demeuré moins célèbre que le texte sur les "hétérotopies », lequel a connu un destin fameux du fait de l'intérêt important qu'il a suscité chez les urbanistes, les architectes, etc., au point qu'une chaire d' "hétérotopologie" sera ouverte par l'architecte Edward Soja à l'université de Californie à Los Angeles dans les années 1980. Le texte sur le corps utopique est donc resté pour une bonne part dans l'ombre - il n'a ainsi pas été repris dans les Dits et Ecrits de Foucault. Comme l'indique Philippe Sabot, cette conférence a largement été délaissée par les commentateurs de Foucault et ce, parce qu'elle «prend clairement l'allure d'une méditation métaphysique sur le corps propre comme fondement d'un rapport originaire à l'espace vécu ${ }^{1}$. Autrement dit, elle peut clairement être lue comme une " approche phénoménologique de l'utopie ${ }^{2}$, à partir de ce qui apparaît comme une "réhabilitation d'une approche phénoménologique de l'être-au-monde $»^{3}$.

Le «corps utopique » débute par une réflexion sur le corps comme lieu absolu. Qu'est-ce que cela signifie ? Le corps est ce lieu que je ne peux simplement abandonner quelque part pour vivre librement mon existence. Il se manifeste dans tout son poids dès le réveil ; chaque matin, il se donne à voir dans la glace comme ce spectacle pénible qu'il me faudra bien emmener partout avec moi. Ainsi que l'écrit Foucault, décrivant l'effet que lui procure la vision de son visage dans le miroir : «Et c'est dans cette vilaine coquille de ma tête, dans cette cage que je n'aime pas, qu'il va falloir me montrer et

\footnotetext{
${ }^{1}$ Cf. Hstl.recherche.univ-lille3.frH ou P. Sabot, « Langage, société, corps. Utopies et hétérotopies chez Michel Foucault », in Materiali foucaultiani, vol. I, n¹ (2012), pp. 17-35.

2 Ibidem.

${ }^{3}$ Ibidem. Sans doute la référence phénoménologique qui inspire Foucault pour ses descriptions est celle de Merleau-Ponty.
} 
me promener $(\ldots) »^{1}$. L'expérience ainsi vécue du corps, c'est celle que décrit alors Foucault par l'expression «topie impitoyable ${ }^{2}$. Face à cette impossibilité de se désolidariser du corps, Foucault montre comment on peut comprendre l'utopie comme « un lieu hors de tous les lieux, mais c'est un lieu où j'aurai un corps sans corps $(\ldots) »^{3}$. C'est-à-dire que l'utopie peut alors être entendue comme ce qui permet d'effacer le corps, de s'en défaire, voire de le transformer dans l'imaginaire. Foucault indique de quelle façon « il se peut bien que l'utopie première, celle qui est la plus indéracinable dans le cœur des hommes, ce soit précisément l'utopie d'un corps incorporel $(\ldots) »^{4}$. Selon cette première lecture, il semble que l'utopie ait d'abord été tournée contre le corps : l'utopie s'épuiserait dans cette possibilité donnée, au sein d'un monde ou d'un espace imaginaire, de biffer le corps, de l'effacer. Et pourtant, comme le montre Foucault par la suite, si l'on s'interroge plus avant sur le lien étroit qu'entretient l'utopie à l'espace, ce n'est pas tant comme espace imaginaire qu'il faut décrire et comprendre d'abord l'utopie. C'est à partir du corps que le lien entre espace et utopie se laisse entrevoir et saisir. De manière plus subtile, le corps recèle une puissance utopique forte qui, après-coup, par la suite seulement, peut donner lieu à une pratique utopisante consistant à effacer le corps.

Mais comment comprendre cette puissance d'emblée utopisante du corps ? Et comment comprendre que c'est comme puissance utopique que le corps peut chercher à se tourner contre lui ? Selon nous, il faut en revenir à l'expérience du miroir. L'expérience du miroir, telle que décrite par Michel Foucault dans « Le corps utopique », possède un statut très particulier. Il faut faire remarquer que dans un premier mouvement de l'analyse, le corps vécu semble d'abord s'identifier à l'image renvoyée par le miroir, comme si se nouait là, dans le reflet de la glace, une image de soi irréductible. Plutôt, comme s'il s'agissait là d'une vision irréductible de soi-même sur laquelle on ne dispose que de peu de pouvoir. Il me faut bien accepter que c'est cette image, ce corps là que je transporte partout avec moi, que je le veuille ou non. Si je suis tentée de fuir, si je ressens le besoin de m'évader, où que ce soit, je dois bien me faire une raison : mon corps est le complice inconditionnel de toutes mes évasions. Pourtant, écrit Foucault, mon corps «ne se laisse pas réduire si facilement $»^{5}$. Qu'est-ce que cela signifie ? Mon corps,

\footnotetext{
${ }^{1}$ M. Foucault, Le corps utopique, op.cit., p. 10.

${ }^{2}$ Ibid., p. 9.

${ }^{3}$ Ibid., p. 10.

${ }^{4}$ Ibidem.

${ }^{5}$ Ibid., p. 12.
} 
malgré ce que m'enseigne l'expérience du miroir, comporte toujours des pans d'invisibilité qu'aucun miroir ne peut capturer. Le miroir semble figer une portion visible de mon corps, sans pour autant pouvoir prétendre l'épuiser totalement. Ainsi que l'écrit Foucault : « Pourtant, ce même corps qui est si visible, il est retiré, il est capté par une sorte d'invisibilité de laquelle jamais je ne peux le détacher $(\ldots) »^{1}$.

Cette invisibilité profonde du corps, cette opacité vertigineuse est précisément ce qui fait du corps un lieu absolu : c'est parce que je ne peux détacher mon épaule de moi-même pour l'inscrire dans un horizon de pleine visibilité, sous mon regard, que le corps est utopique. Si le miroir me renvoie cette visibilité, s'il me permet d'observer des horizons entiers de visibilité, il n'épuise pas toute cette invisibilité vertigineuse de mon corps. Autrement dit, si le miroir possède ce pouvoir de me spatialiser, de me figer quelque part dans un espace délimité, s'il semble donc mettre en suspens ce pouvoir utopique du corps, il ne peut mener cette opération à terme. Cette opération est toujours inachevée. Bien que spatialisé par le miroir, mon corps continue de se donner selon des horizons d'invisibilité que le reflet que me renvoie la glace ne saurait capter. En ce sens, le corps, en tant qu'il est corps utopique, recèle des pans entiers d'opacité qui ne se laissent absolument pas capturer. Comme tels, ces pans de mon corps qui échappent à toute spatialisation, à toute mise en visibilité, sont utopiques dans le sens même défini par Foucault, à savoir : ils sont des lieux sans lieux. En ce sens, il est intéressant de noter que l'épreuve du miroir, décrite sur un mode phénoménologique comme épreuve du corps propre, se voit attribuée un statut particulier et ambivalent par Foucault dans la progression du texte. Le texte s'ouvre en effet sur l'épreuve du miroir comme instrument, d'une certaine façon, de normalisation. Le miroir semble s'apparenter à une métaphore du corps pour autrui sartrien ; « là » aussi autrui est partout, « là » aussi on se voit ou on tente parfois d'apparaître également avec d'autres yeux. Comme le dit très justement Bourdieu dans La domination masculine à propos du miroir: «Instrument qui permet non seulement de se voir mais d'essayer de voir comment on est vu et de se donner à voir comme on entend être vu ${ }^{2}$. Si nous parlons bien d'épreuve à propos du miroir, c'est qu'il est l'horizon où se rencontre et se déploie une pratique subjective d'ajustement, renvoyée sans cesse à son reflet comme à ses échecs, c'est-à-dire, renvoyée à l'impos-

\footnotetext{
${ }^{1}$ Ibid., p. 13.

${ }^{2}$ P. Bourdieu, La domination masculine (1998), Paris, éditions du Seuil, 2014, p. 96.
} 
sibilité d'épouser - ou d'incarner - le regard des autres. « Tous les matins, même présence, même blessure $»^{1}$, écrit Foucault.

De cette façon, l'expérience du miroir est une expérience singulière qui permet de saisir la nécessité et le besoin d'élaborer, pour les hommes, des pratiques utopiques qui consistent à effacer le corps. Pour autant, l'expérience du miroir ne s'épuise pas pour Foucault dans cette nécessité et cette recherche utopique. Plutôt, cette même description de la vue de son corps dans le miroir doit attirer notre attention sur un point ambigu du texte de Foucault, à savoir : c'est l'expérience ou le «stade » du miroir qui semble consister en un moment fondateur, pour le sujet, de la cohésion de son corps comme corps propre. Il faut, sur ce point, revenir à ce qu'écrit Foucault à la fin de son texte. Il écrit ceci :

Après tout, les enfants mettent longtemps à savoir qu'ils ont un corps. Pendant des mois, pendant plus d'une année, ils n'ont qu'un corps dispersé, des membres, des cavités, des orifices, et tout ceci ne s'organise, tout ceci ne prend littéralement corps que dans l'image du miroir ${ }^{2}$.

Ce dernier passage est important car il permet de comprendre comment, à lire Foucault, il n'y a pas à proprement parler de corps (propre) avant l'établissement du stade du miroir. C'est le stade du miroir qui, en spatialisant le corps, joue ce rôle fondamental d'organisation de tous les membres dispersés du petit enfant en un tout cohérent, faisant apparaître l'élaboration et la reconnaissance d'un corps vécu et de son unité. Mais il faut aller plus loin, plus profondément encore : le miroir, par l'acquisition du corps propre qu'il soutient, révèle également le corps comme étant d'emblée utopique. Parce qu'il me spatialise, c'est l'expérience du miroir qui m'apprend également que ce corps fuit de toutes parts et que, en tant que lieu sans lieu, c'est lui qui spatialise tous les objets et toutes les choses qu'il rencontre. Le corps utopique, dans son invisibilité foncière, irréductible, constitue ce point zéro à partir duquel s'organise l'environnement, à partir duquel se déploie chaque chose et se situe chaque objet. De ce fait, le miroir, en me spatialisant, en me situant dans un espace, relativise ce pouvoir utopique, semble le biffer ou le suspendre. Toutefois, comme nous l'avons dit, cet objet aussi singulier qu'est le miroir possède un statut complexe dans l'économie des analyses de Foucault. En me spatialisant, en me donnant la possibilité de me confronter à mon image, à ce reflet que je reconnais comme le mien, ce même miroir

${ }^{1}$ M. Foucault, Le corps utopique, op.cit., p. 10.

${ }^{2}$ Ibid., p. 18. Nous soulignons. 
semble me réduire comme point zéro absolu. Plus que cela, le miroir, rejouant d'une autre façon la situation du corps pour autrui sartrien, participe alors à développer des utopies dont l'enjeu essentiel est de biffer, d'effacer le corps. Effacer le corps, qu'est-ce que cela signifie ici ? Cela ne signifie rien d'autre que de tenter de neutraliser ce corps comme corps toujours situé, comme si je pouvais me transporter ailleurs sans pour autant embarquer mon corps avec moi.

De telles pratiques utopisantes, comme nous le donne à lire Foucault, sont des pratiques qui, même au sein de leur dimension imaginaire, sont spatialisantes. Il s'agit, au sein de telles pratiques, non pas tant de quitter le corps que de lui redonner son pouvoir spatialisant, de point zéro absolu. Il s'agit de retrouver un rapport à l'espace dans lequel c'est le corps qui spatialise les objets et les choses et ouvre l'horizon. En d'autres termes, de telles pratiques ne sont précisément possibles que parce que je suis dotée d'un corps, comme l'expérience ou le «stade» du miroir me l'apprend. Ainsi, comme l'écrit Foucault dans ce très beau passage :

J'étais sot, vraiment, tout à l'heure, de croire que le corps n'était jamais ailleurs, qu'il était un ici irrémédiable et qu'il s'opposait à toute utopie. Mon corps, en fait, il est toujours ailleurs, il est lié à tous les ailleurs du monde, et à vrai dire il est ailleurs que dans le monde. Car c'est autour de lui que les choses sont disposées, c'est par rapport à lui — et par rapport à lui comme par rapport à un souverain - qu'il y a un dessus, un dessous, une droite, une gauche, un avant, un arrière, un proche, un lointain. Le corps est le point zéro du monde, là où les chemins et les espaces viennent se croiser le corps n'est nulle part : il est au cœur du monde ce petit noyau utopique à partir duquel je rêve, je parle, j'avance, j'imagine, je perçois les choses en leur place et je les nie aussi par le pouvoir indéfini des utopies que $\mathrm{j}^{\prime}$ imagine $^{1}$.

C'est sans doute en ce sens que Foucault définit alors le miroir comme une « hétérotopie », c'est-à-dire, comme " un lieu ouvert, mais qui a cette propriété de vous maintenir au dehors $»^{2}$. Il s'agit là, non d'un lieu purement imaginaire, mais de ce que Foucault appelle également une utopie réalisée. Comme l'indique Foucault, il s'agit de lieux bien réels, mais absolument autres, et dont l'emplacement parmi d'autres espaces réels, effectifs, crée des effets de neutralisation des autres espaces, si bien que les hétérotopies sont décrites par Foucault comme des « contre-espaces » ${ }^{3}$. Ce sont

\footnotetext{
${ }^{1}$ Ibid., pp. 17-18.

${ }^{2}$ Ibid., p. 32.

${ }^{3}$ Ibid., p. 24.
} 
des lieux réels, des lieux effectifs, des lieux qui sont dessinés dans l'institution même de la société, et qui sont des sortes de contre-emplacements, sortes d'utopies effectivement réalisées dans lesquelles les autres emplacements, tous les autres emplacements réels que l'on peut trouver à l'intérieur de la culture sont à la fois représentés, contestés, inventés ${ }^{1}$.

Si l'on rapporte à présent la description phénoménologique que livre Foucault du corps utopique aux analyses sartriennes du corps pour autrui et, plus particulièrement, au roman d'Annie Ernaux, la honte apparait comme une expérience où se trouve singulièrement mis à l'épreuve le pouvoir originairement utopique du corps. Minéralisé, c'est comme si le corps, pétri par et dans l'épreuve de la honte, s'était retourné contre lui-même, affectant du même coup sa puissance de spatialisation. Si bien que, comme l'écrit Annie Ernaux, on ne puisse plus l'imaginer ou le sentir. En ce sens, parce que la honte est intimement et éminemment corporelle, c'est encore le corps qui, honteux, cherche à s'évader en tentant de s'effacer. À travers le regard d'autrui, l'expérience de la honte nous confronte à l'épreuve d'une capture des pans de visibilité et d'invisibilité du corps utopique, qui fige son pouvoir de spatialisation. Exproprié d'un point de vue sur lequel il ne peut avoir de point de vue - pour reprendre les mots de Sartre que nous avons cités plus haut , le corps honteux ne semble que pouvoir chercher à se nier pour s'évader, intensifiant et amplifiant toujours plus l'épreuve de la honte. De cette façon, l'essai de Foucault sur le corps utopique permet d'apporter un autre éclairage sur la dimension mystérieuse du corps, sur son pouvoir de spatialisation que le regard d'autrui peut affecter en profondeur. Enfin, on peut faire l'hypothèse que l'imagination recèle ce pouvoir de spatialisation où le corps vécu se trouve pleinement impliqué, rejouant la possibilité d'être le point zéro de ses mondes imaginaires.

Dans ce qui précède, nous nous sommes efforcée d'appréhender le phénomène de la honte comme un phénomène éminemment corporel et social. En mobilisant les descriptions de Sartre sur la visibilité et le corps pour-autrui, celles de Levinas, d'Alain Brossat, d'Agamben et enfin celles d'Annie Ernaux, un des enjeux de notre parcours était de mettre en évidence que pour ressaisir tout ce que la honte a de social, il est nécessaire de la désolidariser du cadre trop restrictif de la faute morale auquel elle est le plus couramment ramenée et ce, en mettant l'accent sur le corps. En ce sens, un second enjeu de nos analyses a consisté dans l'approfondissement des signi-

1 Michel Foucault, cité par P. Sabot, «Langage, société, corps. Utopies et hétérotopies chez Michel Foucault », op. cit., p. 25. 
fications sociales et des valeurs qui se nouent dans les corps dans le cadre des relations intersubjectives. Pour le dire autrement, ce que nous avons voulu souligner, c'est que les projections imaginaires, qui surgissent dans la rencontre des corps et consolident un rapport singulier au corps vécu, surgissent dans un espace pratiquement normé, codifié, qui dépasse, tout en les façonnant d'emblée, les relations intersubjectives. De cet enchevêtrement du monde social et des relations intersubjectives qui s'y déploient tout en le transcendant, l'expérience de la honte, telle qu'elle est notamment décrite par Sartre dans le corps pour-autrui, recèle à notre sens des ressources fécondes pour problématiser une approche phénoménologique du social qui ne céderait pas forcément à la vision intersubjectiviste du monde social - tant décriée par de nombreux commentateurs de Husserl. Enfin, en faisant un ultime détour par «Le corps utopique » de Foucault, nous avons tenté de prolonger notre réflexion sur les rapports entre corps et espace vécus, en suggérant que la puissance utopisante du corps, bien qu'elle puisse être captée par autrui et ainsi être altérée ne peut pour autant l'être totalement.

De la sorte, on peut se demander comment penser ensemble cette puissance utopisante du corps, confrontée à la manière dont le corps propre se trouve pris dans un processus de socialisation, d'incorporation de normes et de valeurs et, enfin, de quelle façon l'image du corps peut être impliquée dans l'imagination. C'est volontairement que nous laisserons cette dernière question ouverte, tout en formulant l'hypothèse que l'imagination puisse consister en une pratique de spatialisation des possibles, où le corps constitue un acteur central.

\section{Bibliographie}

Agamben G., Ce qui reste d'Auschwitz. L'archive et le témoin. Homo Sacer III (1998), trad. fr. P. Alferi, Paris, Éditions Payot \& Rivages, coll. Rivage Poche/Petite Bibliothèque, 2003.

Bourdieu P., La domination masculine (1998), Paris, Éditions du Seuil, 2014.

Derrida J., L'animal que donc je suis, Paris, Galilée, 2006.

Ehrenberg A., Mingasson L. \& Vulbeau A., «L'autonomie, nouvelle règle sociale. Entretien avec Alain Ehrenberg », in Informations sociales, 2005/6, $\mathrm{n}^{\circ} 126$, p. 112-115.

Ernaux A., La Honte, Paris, Gallimard, coll. Folio, 1997.

Fink E., «La nature en nous » (1951), trad. fr. N. Depraz, in Alter, nº 7, 1991, p. 271283. 
Foucault M., Le corps utopique, les hétérotopies, Fécamp, Nouvelles Éditions Lignes, 2009.

Honneth A., "Visibilité et invisibilité. Sur l'épistémologie de la "reconnaissance" », trad. fr. F. Gollain \& C. Lazzeri, in Revue du Mauss, 2004/1, n²3, p. 137-151.

Husserl E., Phantasia, conscience d'image, souvenir. De la phénoménologie des présentifications intuitives. Textes posthumes (1898-1925), trad. fr. R. Kassis \& J.-F. Pestureau, Grenoble, Éditions Jérôme Millon, coll. « Krisis », 2002.

Jamoulle P., Des Hommes sur le fil. La construction de l'identité masculine en milieux précaires, Paris, Éditions La Découverte, 2008.

Le Blanc G., L'invisibilité sociale, Paris, P.U.F, coll. « Pratiques théoriques », 2009.

Levinas E., De l'évasion (1935), Paris, Fata Morgana, 1982.

Richir M., Phantasia, imagination, affectivité. Phénoménologie et anthropologie phénoménologique, Grenoble, Éditions Jérôme Millon, coll. « Krisis », 2004.

Sabot P., «Langage, société, corps. Utopies et hétérotopies chez Michel Foucault», in Materiali foucaultiani, vol. I, n 1 , 2012, p. 17-35.

Sartre J.-P., L'Être et le Néant. Essai d'ontologie phénoménologique (1943), Paris, Gallimard, coll. « Tel», 2013.

Vander Elst M., Entretien avec Alain Brossat. Discussion autour de Autochtone imaginaire, étranger imaginé, Bruxelles, février 2014. Cf. https://www.academia. edu/12479728/Entretien_avec_Alain_Brossat 